\title{
China's national Research Project on WIRELESS SENSOR NETWORKS
}

\author{
Lionel M. Ni AND YunhaO LiU, Shanghai JiaO TONg UniVersity aNd \\ HONG KONG UNIVERSITY OF SCIENCE AND TECHNOLOGY \\ YANMIN ZHU, HONg Kong UNIVERSITY OF SCIENCE AND TECHNOlOgY
}

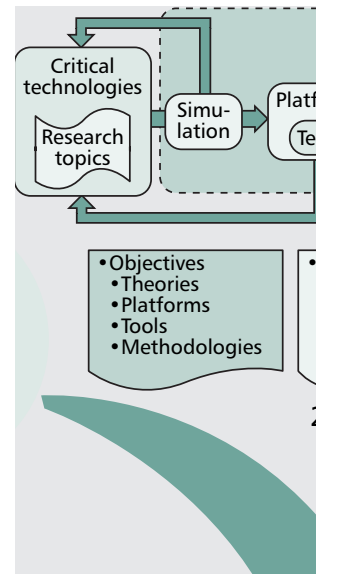

China is experiencing rapid economic growth, but is also facing serious social and environmental problems. The advance of WSNs has made it possible to deploy low-cost networked sensors to address many huge challenges.

\begin{abstract}
China is experiencing rapid economic growth, but is also facing serious social and environmental problems, such as safety threats in the mining industry, environmental pollution, and transportation congestion. The advance of wireless sensor networks (WSNs) has made it possible to deploy low-cost networked sensors to address many huge challenges. A national research project, supported by the prestigious National 973 Program of China, was launched in September 2006. The project adopts an application-driven methodology and aims to address the real-world critical problems facing Chinese society. This project will follow a systematic approach, integrating technologies as a system solution to critical problems. Although this is a basic research project, the project has an ambitious goal of solving practical problems with the potential of commercialization. The success of this project will bring significant benefits to China's sustainable economic and social development.
\end{abstract}

\section{INTRODUCTION}

China, the most populous developing country, has been boosting its economy in the last two decades. The standard of living has been raised noticeably, and the rapid growth of the economy has gained wide recognition in the world. Aside from the significant achievements made by China, however, a number of serious social and environmental problems have emerged. Among others, the following three salient problems are critical for the healthy development of China.

Safety threats in the mining industry. Stimulated by the rapid economic development, the demand for energy, such as gas, oil, and coal, has been increasing more than ever. Therefore, the coal mining industry flourishes, and coal mines have spread widely throughout China today. However, many coal mines, especially those privately operated, have poor safety conditions. Serious accidents, such as explosions of gas or dust and inundations, are reported constantly, causing many fatalities every year. It is reported that the number of fatalities in coal mines in Inner Mongolia province alone was as high as 160 in 2003.

Environmental pollution. Polluted air, water, and soil are critical problems in many places in China. Rivers are being polluted by various sources, such as city sewage, factories, agricultural runoff, and mining activities. The pollution of water resources can have severe and long-term effects on the environment and human health. In 2005 the explosion of a petrolic chemical factory caused a large leakage of poisonous chemical pollutants. The Songhua River, the main source of drinking water for millions of residents in Northeast China, was badly polluted by the leakage. The water provision of Harbin, the capital of Heilongjiang province with a population of 9 million, was suspended for more than 10 days. This accident also affected residents along the downstream part of this river in Russia.

Transportation congestion. As more and more people possess private cars, roads are becoming increasingly crowded, especially in metropolises during rush hour. Although infrastructure construction continues, the urban population suffers from traffic jams every day. In Beijing it often takes a car one hour to drive from home to the office during rush hour, even when the distance is no more than $20 \mathrm{~km}$.

Recent advances in electronics, embedded systems, and wireless communications have made it practical to develop low-cost and lowpower wireless sensor networks (WSNs) for realtime monitoring of various environments of interest. WSN technology has attracted tremendous interest from academic circles, industry, and government due to its unique potential as a cost-effective solution to a wide spectrum of real-world challenges. The advance of WSN techniques makes it possible to address many of the huge challenges through pervasive and automated information collection from any site with which people are concerned.

In contrast to traditional wired computer networks, WSNs display many unique characteristics. Tiny sensors are usually subject to stringent 
resource constraints, such as limited memory, computing power, and bandwidth. A sensor node usually does not have a globally unique identification (ID) because such an ID is often too costly for tiny sensors. More importantly, a tiny sensor is typically powered by a small battery, implying that the lifetime of an individual node is short. A sensor system usually consists of a large number of sensors being deployed in a remote and untethered field. Such a system is often unattended and subject to frequent unexpected node failure. The deployment of a sensor system can be random, for example, sprayed from a flying aircraft, resulting in irregular topologies.

These unique characteristics clearly distinguish WSNs from traditional networks and make most conventional technologies inappropriate for sensor network design. In response to serious challenges raised in WSNs, extensive research efforts have been made. Many research projects have been performed in China and worldwide. Many aspects have been investigated, such as localization [1], time synchronization [2], in-network information processing [3], event detection [4], and routing algorithms [5]. A proposed annual event, the Chinese Conference on Wireless Sensor Networks, received increased attention regarding this emerging technology from Chinese academia and industry. You can visit http://www.sensornet.org.cn/cwsn/2007/default.ht $\mathrm{m}$ for details of the sessions held during the first conference in September 2007.

Extensive existing work on sensor networks certainly is a good starting point for us to address the huge problems in China. Existing approaches, nevertheless, are far from adequate considering the unique characteristics exhibited by our targeted application scenarios. First, typical application fields, for example, the urban area of a city, can be immense, inevitably requiring large-scale deployment of sensor nodes. This strongly suggests that the node platform should not be merely power-efficient but also cost-effective. Otherwise, the high cost of the system will prevent practical deployment in large-scale applications. Second, the applications, such as mine tunnels monitoring and chemical pollution detection, are particularly mission critical. Improper behavior of the system may lead to severe fatalities. Thus, it has become mandatory to design and implement a reliable, robust, and secure system. Finally, almost every sector of the society and economy is suffering from the wide range of issues caused by these huge and challenging problems. This implies that a proposed solution should be exploited as soon as possible. It certainly requires extensive experiments and verification of the sensor system as a whole in real-world environments that may vary from location to location. This unique product-oriented philosophy poses rigid requirements for the solutions to be developed.

We have identified the pitfalls associated with existing projects. On the one hand, they suffer from the lack of a systematic study on the sensor network system. Each of them typically focuses on a small aspect and proposes an isolated solution for a certain scenario. Obviously, a system integrated by multiple isolated solutions may not be optimal. Even worse, those isolated solutions may conflict with each other when they are put together as a whole solution. On the other hand, few projects were proposed especially for large real-world challenges. Designed systems are rarely tested in a running application environment for a reasonably long time. A small testbed or simplified simulators usually are employed instead. It is not sufficient when such a system must be a large-scale deployment or even commercialized.

In response to the limitations with existing projects and the urgent needs from real-world challenges that exist in China today, we proposed an innovative research project on WSNs. It is a collaborative effort among ten renowned universities and institutes in China, including Shanghai Jiaotong University, Hong Kong University of Science and Technology, University of Science and Technology of China, Nanjing University, Zhejiang University, Harbin Institute of Technology, Tsinghua University, National University of Defense Technology, Institute of Computing Technology, and the Institute of Software in Chinese Academy of Sciences. The goals of this project are as follows.

- To develop a cost-effective and low-power versatile sensor network platform for various application environments.

- To design a set of core energy-efficient protocols that can be integrated together for delivering optimized solutions to various applications. Such protocols should be open source.

- To construct a large-scale testbed, in combination with effective evaluation methodologies, enabling a precise performance study of protocols or systems to be developed in diverse physical environments.

- To address several critical problems facing the nation with effective low-cost sensor network solutions. We aim at full commercialization of our advantageous solutions.

This project is supported by the National Basic Research Program of China (also known as the 973 Program), which was established in September 2006 with the project title "Research on Fundamental Theories and Critical Technologies for Wireless Sensor Networks." The prestigious 973 Program, initiated in June 1997, is China's on-going effort to further science and technology. It is administrated by the Ministry of Science and Technology. Aimed at the major strategic needs of the nation, it supports innovative studies of scientific issues relating to the sustainable development of the society. This project is deemed as part of the implementation of the national strategies on environment, energy, natural resources, and information technology. In line with the eleventh five-year plan of national economic and social development, this project will contribute to building a resource-conserving and environment-friendly society.

The rest of the article is structured as follows. We first give an overview of this national project. We then introduce the progress that we have made thus far. Next, we outline the future research directions. Finally, we conclude this article.
In response to the

limitations with exist-

ing projects and the

urgent needs from

real-world challenges

that exist in China

today, we proposed

an innovative

research project on

WSNs. It is a

collaborative effort

among ten

renowned

universities and

institutes in China. 
This project adopts

an application-driven

methodology and tightly focuses on the real needs of

Chinese society.

In addition, it

follows a systematic approach throughout the project.

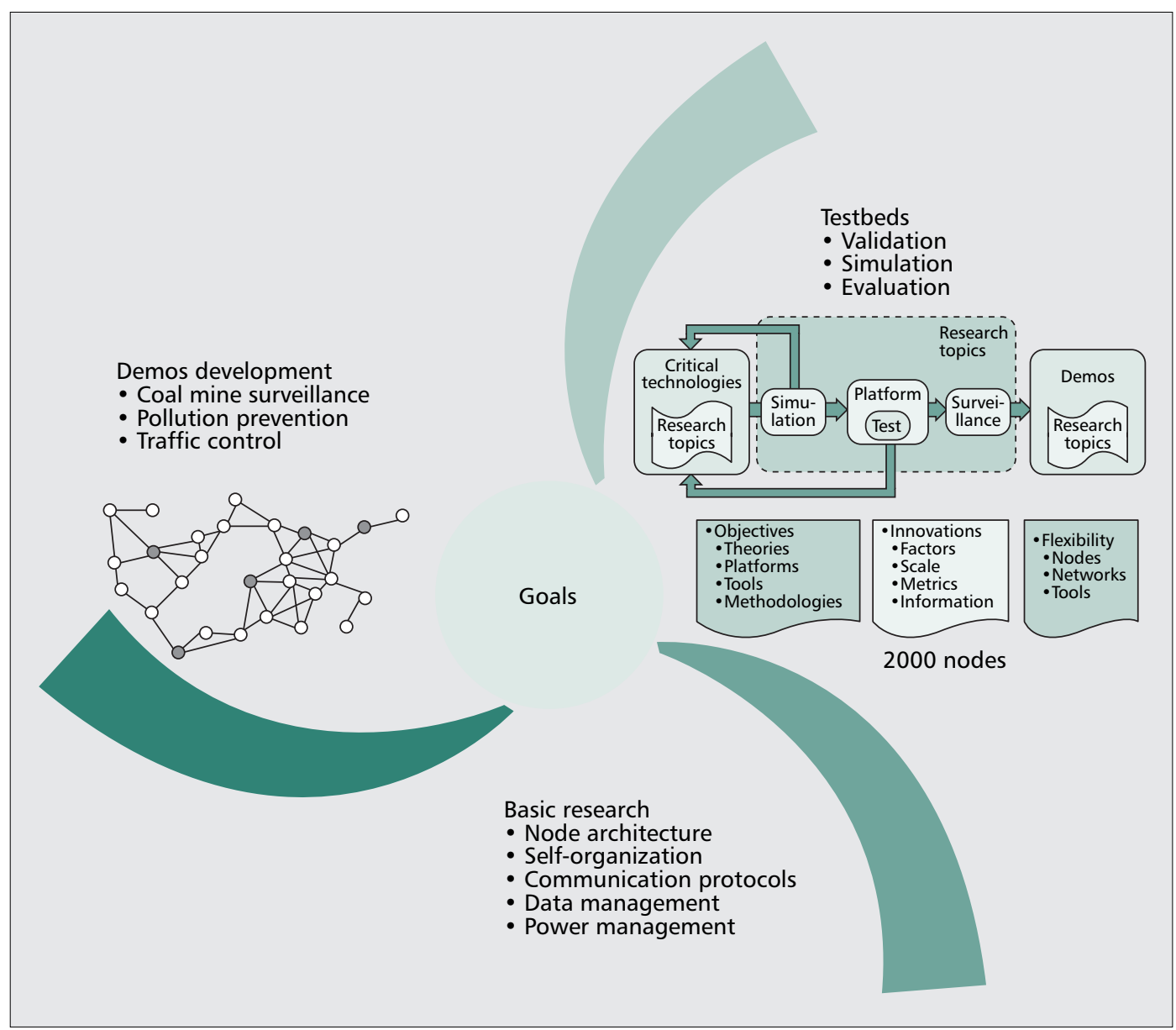

Figure 1. Logical organization of the national project.

\section{Project OVerview}

This project mainly consists of three components that are centered around the goals of the project: demonstration development, basic research, and testbed construction. The logical organization of the project is illustrated in Fig. 1.

This project adopts an application-driven methodology and tightly focuses on the real needs of Chinese society. In addition, it follows a systematic approach throughout the project, that is, it will integrate technologies that are to be acquired in the project as a whole system in addressing the challenges. We will develop advanced solutions for three killer applications.

- Coal mine surveillance. The threats faced by coal miners are fire, explosion, inundation, and falling of high walls and roofs. Real-time surveillance of critical environmental conditions, such as gas, dust, oxygen, and water is crucial. We will design an integrated surveillance system to improve the state-of-the-art of safety technologies for coal mines.

- Water pollution monitoring. We will develop sensor monitoring systems to tackle water pollution that will advance the water pollution prevention technologies in China. In general, pollution sources are geographically dispersed over a vast area. It is difficult for human inspectors to discover all sources, especially some hidden points of pollutant discharge. In addition, when serious pollution occurs, it is of great importance to monitor the water quality in real-time and report dangers in a timely manner.

- Urban traffic monitoring and control. We will develop sensor systems for monitoring traffic information for city roads and help the transportation administration make better decisions in conducting fine-grained control of traffic. Traffic information in a city is by nature dynamic. There is no cost-effective automated approach for traffic monitoring today. The system to be developed will provide a low-cost solution to real-time monitoring of traffic information.

Centering on the three killer applications, Fig. 2 depicts the project architecture, in which tasks are grouped into work packages (WPs).

In WP1, we will develop a node platform. Taking advantages of existing node platforms, such as Berkeley MICA2, the node platform also will explore possible design alternatives and add advanced features such as better memory management and event scheduling. Low-power wireless communication technology (e.g., IEEE 802.15.4) will be employed.

WP2 will develop essential core protocols for self-organization and self-configuration of a sensor network. Important protocols include distributed localization, time synchronization, topology control, and power management. In the process of protocol designs, we will pay special 
attention to the compatibility and optimization of different protocols to work together smoothly.

In WP3, we will devise power-efficient communication protocols, such as medium access control (MAC) protocols and routing algorithms. We must develop a number of different routing algorithms because in different application scenarios, data flow patterns can be very different. For query dissemination and network reprogramming, the data are propagated from the sink to sensors. For regular data collection applications, the dominant data are from sensors to the $\operatorname{sink}(\mathrm{s})$. In some cases, we also may want a sensor to deliver a packet to one or multiple sensors.

WP4 will focus on mechanisms for connecting a sensor network to the Internet so that users are able to access the data available in the sensor network with an option of having multiple sinks. This can greatly increase data accessibility of the sensor network and empower richer interactions of the sensor system with other applications.

In WP5, we will develop data management techniques for sensory data. Because tiny sensors are resource-constrained, it is unwise to route all raw data back to the gateway. Therefore, in-network processing techniques, such as fusion or aggregation, are vital for energy conservation in sensor networks. We also will develop database techniques allowing users to query a sensor network using interfaces similar to operating traditional databases.

Based on system solutions to the three killer applications as stated previously, WP6 will further develop various demos, which are very important to enable our solutions and technologies to have the potential for commercialization. To realize our ambitious goal of commercializing our technologies, the demos must display the full advantage of WSN-based system solutions.

To facilitate the entire research and development for the project, in WP7 we will construct a large-scale testbed (with more than 2000 nodes). Along with the testbed, we also will design evaluation and diagnostic techniques.

\section{ReseARCh Progress}

In this section, we briefly introduce the research progress that we have made. Since the establishment of the project, we have conducted a number of successful research studies. In WP1, we designed a new node platform that features lowpower consumption and rich interfaces for sensing devices. In addition, we are making efforts on developing cost-effective sensors to meet our project requirements. We have produced an ultrasonic sensor and an infrared sensor that feature high sensitivity in detecting moving objects that can be used widely in object-counting scenarios (our team won second place at the 2007 Information Processing in Sensor Networks Extreme Sensing Competition). We also are making progress in designing small chemical sensors that can be utilized in water pollution monitoring.

We designed a rich set of integrated core protocols that are not specific to an application. Such a protocol easily can be plugged into an

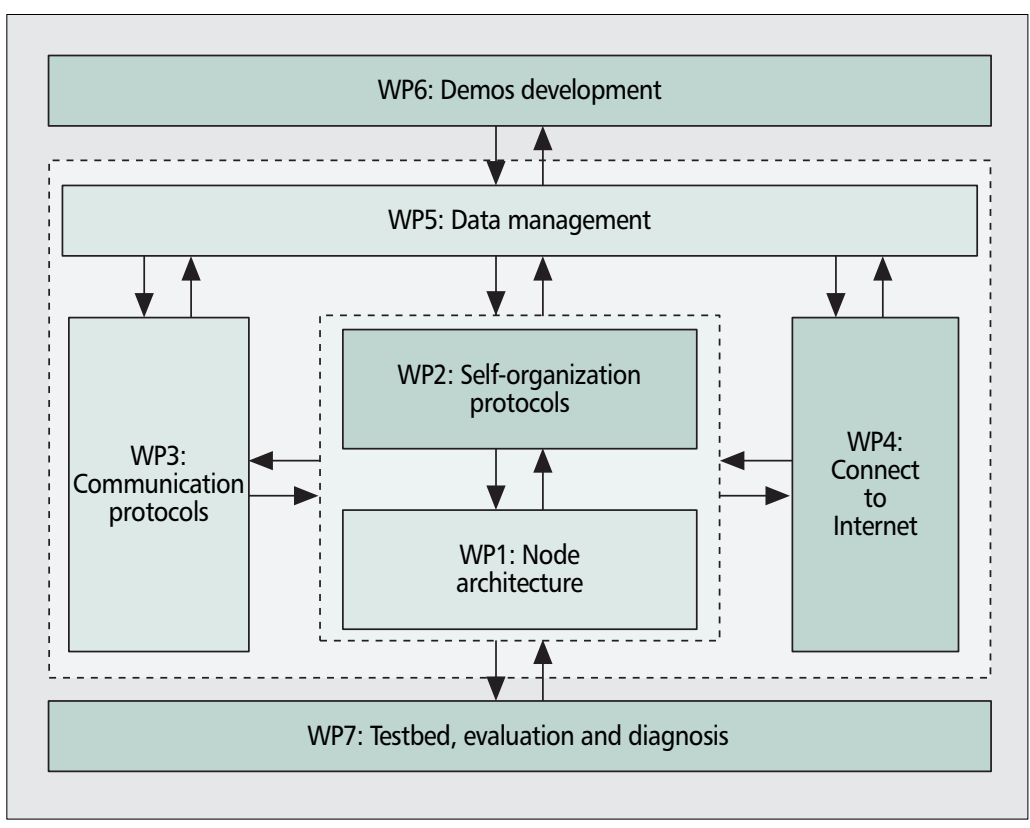

Figure 2. Work package perspective of the project architecture.

application when the function provided by this protocol is desired in this application. We have put particular emphasis on the compatibility of different protocols during the design of these protocols. When two or more protocols are integrated in the same application, the system should work well and energy efficiency is conserved.

In WP2, an effective topology control algorithm [6] has been devised for 802.15.4-based sensor networks. Network connectivity is guaranteed when as many sensor nodes as possible are put into power-save mode for a greater degree of energy conservation. We proposed a fully localized ID assignment algorithm called selforganized ID assignment (SIDA). SIDA presents great potential for scalability, flexibility, and energy efficiency by enabling online ID assignment and various optimizations according to different communication paradigms [7]. Localization is crucial for most sensor network applications, and range-free approaches are superior to range-based ones because of lower cost and higher robustness. However, existing rangebased algorithms fail in anisotropic sensor networks without a large number of known-location seeds. We have recently proposed the first solution [8] to achieve accurate localization in anisotropic sensor networks with only three seeds. Transceiver-free object tracking [9] can accurately locate mine workers even when the workers are poorly equipped or have no radio devices.

Clustering is crucial for many operations in a sensor network, such as MAC, routing, and data aggregation. In WP3, we designed a distributed clustering algorithm [10] that groups sensor nodes into clusters when the maximum hop distance from a member node to the cluster head is given. This algorithm can evenly distribute clusters over the network while minimizing the cluster number and traffic overhead.

In WP7, we constructed a medium-sized testbed with more than 200 nodes. In addition, 
As basic research, we will continue our efforts to build a low-power and cost-effective node plafform. We will improve the node design based on our extensive tests and usage in practice. In addition, we also will study the limitations of Tiny $0 S$. we also developed an emulation system [11] that enables realistic performance evaluation for sensor network applications. Performance evaluation based on testbeds of a large number of sensor nodes is subject to limitations of high cost, poor maintenance, and limited controllability. To complement testbeds, we developed a PC-based emulation environment in which applications can be directly run for debugging, testing, and performance evaluation. Additionally, studies on the lower layers (e.g., hardware drivers, OS, and networking), as well as crosslayer techniques also can be performed in this environment by plugging the target modules into the emulator.

Because the project is highly application-driven, our research activities centered on the solution development of several killer applications that are highly desirable for the sustainable development of China. In WP7, we have developed preliminary but exciting sensor systems for solving large-scale real-world problems.

In the mining industry, it is common practice that a mine tunnel stretches underground as deep as several hundred meters and as long as several kilometers. We have closely cooperated with the S. H. Coal Mine Co. in the province of Inner Mongolia in North China. We conducted a field study of mine tunnels and found that the safety of mining workers is highly subject to threats of structural collapses. Most disasters are the result of small collapses caused by falling roofs or walls. We designed a structure-aware, self-adaptive WSN system, called SASA [12]. It can quickly detect the collapsed hole regions and accurately provide the location information of collapsed regions to control centers and nearby mine workers. This is particularly valuable to enable timely withdrawal of the workers to save their lives.

In China, the majority of the lakes are severely polluted by various kinds of factories. It is useful to study the pollution distribution in a lake and to implement countermeasures. Contour mapping has been widely recognized as a comprehensive method to obtain distribution information. A straightforward approach for contour mapping is to collect sensory data from all the sensors in the monitored field and then construct the contour map at the sink. However, delivering a huge amount of data back to the sink incurs heavy traffic, which rapidly depletes the energy of sensor nodes. To address the inherent limitations of existing approaches, we proposed a contour map-based event detection [13] to detect complex events (with spatial and temporal constraints) that often appear in mine tunnels. We also proposed Iso-Map [14]. By intelligently selecting a small portion of the nodes to generate and report data, Iso-Map can construct high fidelity contour maps while significantly reducing network traffic and computational overhead.

We have extended our research to the sea surface, which also suffers constant pollution. We explore the possibility of deploying networked sensors on the sea surface. Sensor nodes are anchored to the sea bottom, floating within a restricted area, which we call restricted floating sensors (RFSs). We started with investigating the positioning problem of sensor nodes on a sea surface. It is apparent that existing approaches for mobile sensor networks cannot directly be applied to the RFS network, because they do not capture the special nature of the RFS network. We constructed a tailor-made network model for this unique network and proposed an effective algorithm to locate the dynamic location of each sensor [15]. The experiments with a prototype system on the sea surface have revealed promising results and demonstrated that our approach is viable and efficient.

Shanghai, the largest metropolis in China, is facing the escalating challenge of traffic congestion. The Shanghai government has launched the Shanghai Transportation Grid project, which has a long-term goal of building a metropolis-scale transportation information system with pervasive information collectors, such as sensors and radio-frequency identification (RFID) readers. In this system, a fundamental service is to quickly locate a given vehicle. Network-wide location update enables instant query response but introduces overwhelming network traffic overhead. We have proposed a novel real-time vehicle-tracking protocol for the large-scale gird system. For each vehicle, an advantageous hierarchy over the overlay network of local nodes is constructed and dynamically maintained while the vehicle is moving. The system can track any vehicle in the system in real-time while introducing modest network traffic overhead.

In addition, we also designed several generic algorithms, such as object tracking, geocasting, and event detection that can be used in a wide variety of applications. They also are very useful in the three identified killer applications. For details of our research publications and research activities, please visit our Web site at http://wsn.973program.org/.

\section{FUTURE WORK}

We have described a five-year on-going active project. With our ambitious goals in mind, we will conduct more research studies in the future. In this section, we briefly outline the research directions.

As basic research, we will continue our efforts to build a low-power and cost-effective node platform. We will improve the node design based on our extensive tests and usage in practice. In addition, we also will study the limitations of Tiny OS, which is currently the most popular operating system for sensor nodes. Advanced features, such as better event scheduling, will be added to facilitate application development for critical real-world challenges.

Regarding testbed construction, we gradually will add more nodes to the testbed and also design a set of effective methods for evaluating algorithms and protocols. The objectives are easy deployment of the testbed and strong capability for emulating diverse physical environments. Additionally, users must be able to easily collect data that characterize an experiment that later can be used to precisely study the performance of the tested protocol. To this end, we plan to design powerful data collectors, which 
are placed particularly in the testbed for data collection purposes.

The task of solving real-world problems, especially the three identified as critical ones, will remain the focus of this project. Our strategy is to closely collaborate with the stakeholders, such as factories, volunteer organizations, and local government in the process of addressing the problems by developing advanced sensor network solutions.

In coal mine surveillance scenarios, we found that $3 \mathrm{D}$ development of sensor networks is required for accurate monitoring. Previous work usually claims that a solution designed for $2 \mathrm{D}$ environments can be easily extended to 3D environments. Our experiences, however, have revealed that $3 \mathrm{D}$ environments display much more complex dynamics and pose barriers for effectively representing higher dimensional sensory data. We will focus on developing distributed protocols for 3D environments, such as routing algorithms and aggregation techniques.

For water pollution prevention, we will focus on monitoring the water quality of rivers. A river can span a vast area with complicated geographical features. Pollution sources may be scattered unpredictably along the river. Dense deployment of sensors along the whole river is difficult, if not impossible. We need effective, self-configuring, and distributed algorithms for such unique application environments.

For traffic monitoring and control in metropolises, we will focus on distributed traffic information collection and processing to enable better decision making for road plans and transportation regulations. Due to the huge amount of data streams, it is impossible to collect all the data into a centralized unit where data analysis is performed. Distributed cooperative processing techniques on local raw data streams are of great importance. Data mining methods also are required for gathering valuable information from the huge volume of data.

\section{CONCLUSIONS}

China is experiencing rapid economic growth, as well as facing many serious social and environmental challenges. Wireless sensor network technology is very promising as a low-cost solution to a wide array of real-world challenges. We proposed an innovative project on networked sensor systems that has gained the support of the central government (the 973 Program under Grant No. 2006CB303000). The project adopts an application-driven methodology and aims to address the real-world critical problems facing Chinese society. We have performed many indepth research studies. This article has introduced the national project on wireless sensor networks. We have reported our progress and discussed future directions. We believe that the success of this project will bring tremendous benefits to economic and social development in China.

\section{REFERENCES}

[1] A. Savvides, C. Han, and M. Srivastava, "Dynamic Fine Grained Localization in Ad-Hoc Sensor Networks," Proc. ACM MOBICOM, Rome, Italy, 2001.

[2] W. Su and I. Akyildiz, "Time-diffusion Synchronization Protocol for Wireless Sensor Networks," IEEE Trans. Net., vol. 3, 2005.

[3] S. Madden et al. "TAG: A Tiny Aggregation Service for Ad Hoc Sensor Networks," Proc. USENIX OSDI, 2002.

[4] Y. Zhu et al., "Low-Power Distributed Event Detection in Wireless Sensor Networks," Proc. IEEE INFOCOM Minisymposium, 2007

[5] C. Intanagonwiwat, R. Govindan, and D. Estrin, "Directed Diffusion: A Scalable and Robust Communication Paradigm for Sensor Networks," Proc. ACM MOBICOM, Boston, MA, 2000.

[6] J. Ma et al., "Energy-efficient Localized Topology Control Algorithms in IEEE 802.15.4-based Sensor Networks," IEEE Trans. Parallel and Distrib. Sys., 2007.

[7] J. Lin, Y. Liu, and L. M. Ni, "SIDA: Self-Organized ID Assignment in Wireless Sensor Networks," Proc. IEEE MASS, 2007.

[8] M. Li and Y. Liu, "Rendered Path: Range-Free Localization in Anisotropic Sensor Networks with Holes," Proc. ACM MOBICOM, 2007.

[9] D. Zhang et al., "An RF-based System for Tracking Transceiver-free Objects," Proc. IEEE PerCom, 2007.

[10] Q. Chen et al., "An Energy-efficient K-hop Clustering Framework for Wireless Sensor Networks," Proc. 4th Euro. Conf. Wireless Sensor Networks, 2007.

[11] H. Wu et al., "VMNet: Realistic Emulation of Wireless Sensor Networks," IEEE Trans. Parallel and Distrib. Sys., vol. 18, 2007, pp. 277-88.

[12] M. Li and Y. Liu, "Underground Structure Monitoring with Wireless Sensor Networks," Proc. IPSN, 2007.

[13] W. Xue et al., "Contour Map Matching For Event Detection in Sensor Networks," Proc. ACM SIGMOD, 2006.

[14] Y. Liu and M. Li, "Iso-Map: Energy-Efficient Contour Mapping in Wireless Sensor Networks," Proc. IEEE ICDCS, Canada, 2007

[15] Z. Yang, M. Li, and Y. Liu, "Sea Depth Measurement with Restricted Floating Sensors," Proc. IEEE RTSS, 2007.

\section{BIOGRAPHIES}

LIONEL M. Nı [F] (ni@cse.ust.hk) earned his Ph.D. degree in electrical engineering from Purdue University in 1980. He is Chair Professor at the Hong Kong University of Science and Technology (HKUST) and Distinguished Professor at Shanghai Jiao Tong University. He is chief scientist of the National 973 Program on wireless sensor networks, director of HKUST MOE/MSRA IT Key Lab, and director of HKUST Digital Life Research Center.

YUNHAO LIU [SM] (liu@cse.ust.hk) received a B.S. degree from Tsinghua University, an M.A. degree from Beijing Foreign Studies University, and M.S. and Ph.D. degrees in computer science and engineering from Michigan State University. He is now an assistant professor in the Department of Computer Science and Engineering at HKUST. His research interests include pervasive computing, sensor networks, and peer-to-peer computing.

YANMIN ZHU (zhu@cse.ust.hk) received his B.E. degree from the Department of Computer Science at Xi'An Jiao Tong University in 2002 and his Ph.D. from the Department of Computer Science and Engineering at HKUST in 2007. He is working as a research associate in the Department of Computing at Imperial College London. His research interests are wireless sensor networks and distributed computer systems.
Distributed coopera-

tive processing

techniques on local

raw data streams

are of great

importance.

Data mining

methods also are

required for

gathering valuable information from the huge volume of data. 